\title{
Comprehensive Evaluation Model of Toll Plaza System
}

\author{
Liyao Li \\ School of North China Electric Power University, Baoding 071000, China \\ Ilyhbdl@163.com
}

Keywords: Toll plaza, Evaluation model, Delay time, Accident rates.

\begin{abstract}
In this paper, a comprehensive evaluation model (CEM) is proposed to verify the superiority of a new solution compared with the common toll plaza schemes. Targeting on the features of the evaluation system, dimensionless method has been carried out to analyze the indexes, which are total delay times, the accident rates, the cost and the traffic flow density. The weight of different indexes is depended on the decision-maker. Using the CEM, we can get a score of the new solution. The lower score represent the better performance.
\end{abstract}

\section{Introduction}

It is well known that unreasonable road design and disordered traffic conditions are important factors in triggering accidents. As a part of highway design, tollbooth has close relationship with road safety, and there are many security problems in actual operation and management [1]. Time is equally important and N.Gans et al. [2] emphasized the importance of service time. Therefore, making the vehicles efficiently and orderly go through the tollbooths can reduce people's waiting time and the occurrence of road accidents. A toll plaza consists of the "fan-out" area before the barrier toll, the barrier toll itself, and the "fan-in" area after the toll vividly. The "fan-in" area after the toll is easy to cause traffic jams since the vehicles entering less lines, more seriously, accidents occur sometimes. So determine the shape, the size, and the merging pattern of the area following the toll barrier scientifically is very important and necessary. Establishing the evaluation model to determine the optimal solutions (the shape, the size, and the merging pattern of the area following the toll barrier) is the most critical issue of the problems. Important considerations should be incorporated in the model above are accident prevention, throughput, and cost.

\section{Definition of parameters}

\subsection{The total delay time}

The process through toll plaza can be divided into three stages: entering the transition section, waiting for the service section and leaving the transition section. Each phase corresponds to a time, so the total time is:

$$
T=T_{n}+T_{w}+T_{u}
$$

Where $T_{n}, T_{w}$ and $T_{u}$ respectively represents the average time consumed by the vehicle flow entering the transition section, waiting for the service section and leaving the transition section. $T$ is the total average delay time consumed by the vehicle flow through the toll plaza.

\subsection{The accident rates}

Z.Z. Zhang found that $x_{m}$ has no influence on $\lambda_{\text {re }}$ basically [3]. He established a rear-end accident prediction model (Eq.3) based on the statistical analysis on the rear-end collisions and the traffic flow parameters of the confluence area. ( $A, B, C$ and $D$ are coefficients and $\psi$ is a constant)

$$
\lambda_{\mathrm{re}}=\exp \left(\psi+A x_{q}+B x_{b}+C x_{v}+D x_{h}\right)
$$

Similarly, the land-change accident model can be listed by the follows, $\lambda_{l c}$ has a relationship with all these five factors: [3] ( $P, Q, R, S$ and $T$ are coefficients and $\zeta$ is a constant)

$$
\lambda_{l c}=\exp \left(\zeta+P x_{q}+Q x_{b}+R x_{v}+S x_{h}+T x_{m}\right)
$$


According to the above analysis, the total traffic accident prediction model of freeway "fan-in" area is as follows: [3]

$$
\begin{aligned}
& N_{\mathrm{m}}=\lambda_{\mathrm{re}}+\lambda_{l c}=\exp \left(\psi+A x_{q}+B x_{b}+C x_{v}+D x_{h}\right) \\
& +\exp \left(\zeta+P x_{q}+Q x_{b}+R x_{v}+S x_{h}+T x_{d}\right)
\end{aligned}
$$

The total traffic accidents in toll plaza is composed by land-change accidents and rear-end accidents. Define the accident rate $f$ as traffic accident index, which can reflect the security of the designed plaza. And the accident rates can be described as:

$$
f=100 \times \frac{N_{m}}{q_{1}}
$$

Where, $f$ notes accidents per hundred vehicles (times/one hundred vehicles), $N_{m}$ notes the sum of the rear-end accidents and land-change accidents, $q_{l}$ notes the throughput ( $\mathrm{pcu} / \mathrm{h}$ ).

Table 1 Symbols and Explanations

\begin{tabular}{cc}
\hline Symbols & Explanation \\
\hline$x_{q}$ & Throughput $(\mathrm{pcu} / 0.5 \mathrm{~h})$ \\
$x_{b}$ & Proportion of large vehicles \\
$x_{\mathrm{v}}$ & Average difference between running vehicles $(\mathrm{km} / \mathrm{h})$ \\
$x_{\mathrm{h}}$ & Average headway between vehicles $(\mathrm{s})$ \\
$x_{\mathrm{m}}$ & the ratio of the combined traffic to the total traffic (i.e. the confluence ratio ) \\
$\lambda_{\mathrm{re}}$ & the number of rear-end accidents prediction \\
$\lambda_{\mathrm{lc}}$ & the number of land-change accidents prediction \\
\hline
\end{tabular}

\subsection{The cost}

We choose the symbol $C$ to represent the cost of the land and road construction of the toll plaza. Because the cost and the area size are positively correlated, we use the area size $(s)$ to present the cost $(C)$ degree roughly, that is:

$C \propto s$

\subsection{The traffic flow density}

Since use the throughput $q_{1}$ to analyze the problem is inaccurate, divide the throughput $q_{1}$ by the area size s can get a new index- the traffic flow density.

$$
\rho=\frac{q_{1}}{s}
$$

Where the units of each factors are: $q_{1}-\mathrm{pcu} / \mathrm{h}, s-\mathrm{m}^{2}, \rho-\mathrm{pcu} / \mathrm{h} / \mathrm{m}^{2}$.

\section{The establishment of Comprehensive Evaluation Model}

When the following inequality (Eq.8) is set up, we think that our solution is better than the ordinary one or current one.

$$
\alpha \frac{T^{\prime}}{T}+\beta \frac{f^{\prime}}{f}+\gamma \frac{C^{\prime}}{C}+\delta \frac{\rho^{\prime}}{\rho}<\alpha+\beta+\gamma+\delta
$$

Where

$T, f, C$, and $\rho$ are the factors value calculated of the analytic toll plaza;

$T^{\prime}, f^{\prime}, C^{\prime}$, and $\rho$ 'are the factors value calculated of the improved solution of the analytic toll plaza;

$T^{\prime} / T, f^{\prime} / f, C^{\prime} / C$ and $\rho / \rho$ are dimensionless processing;

$\alpha, \beta, \gamma$, and $\delta$ are weighing factors:

when $\alpha: \beta: \gamma: \delta=1: 1: 1: 1$ means the design goal requires the balance of the four indexes.

When $\alpha: \beta: \gamma: \delta \neq 1: 1: 1: 1$,

For example:

when $\alpha: \beta: \gamma: \delta=2: 1: 1: 1$ means the design goal is the pursuit of the smallest $T$.

when $\alpha: \beta: \gamma: \delta=2: 2: 1: 1$ means the design goal is the pursuit of the smallest $T$ and $f$, and $T$ and $f$ are equally important. 


\section{The Sensitivity Analysis of $f$}

Similarly, we define $x_{b 1}$ as the proportion of large vehicles after using dedicated lane for large vehicles in our solution and define: (Eq.9)

$$
x_{b 1}=v x_{b}
$$

Where $v$ is a correction coefficient when use dedicated lane for large vehicles. The proportion of large vehicles merging the main lane will use the dedicated lane, which will decrease the accident, so the range of $v$ is $0<v<1$. We take $v$ is 0.5 before roughly before. Fig. 1 shows the sensitivity of $T$ with $x_{b 1}$ changing.

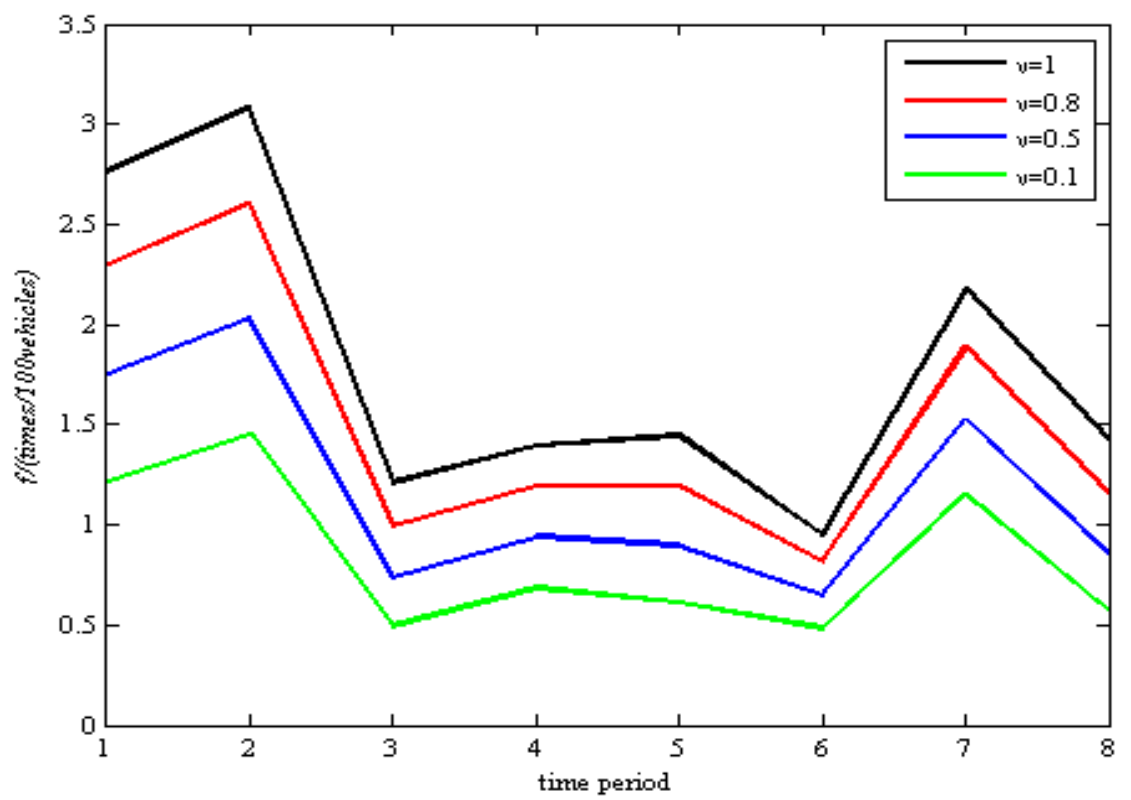

Fig. 1 the sensitivity of $\mathrm{f}$ with q11 changing $\mathrm{xb} 1$

We find that the accidents per hundred vehicles $f$ changed obviously in Fig.1 when we choose $v$ is $0.1,0.5,0.8$ and 1 respectively. So the accidents $f$ is sensible to $x_{b 1}$.

\section{Analysis of the model}

Obviously, the smaller values of $T, f$, $C$ reflects the improvement, but we cannot explain the value of $\rho$ is larger or smaller relatively. It should be noted that, decision makers should measure the value of $\rho$ of the areas before evaluating the solution because we do not know the value of $\rho$ is too great or too little. For example, if the area traffic is very crowded, which means the value of $\rho$ is too great, so decreasing the value the four indexes is the solution's aim. But if the area traffic is far from crowded, which means the value of $\rho$ is too little, so we can increase the value of $\rho$. Under these circumstances, we are no longer use the Eq.8, so we use $1 / \rho$.instead of $\rho, 1 / \rho$ '.instead of $\rho$ ', then we obtain the following equation. (Eq.10)

$$
\alpha \frac{T^{\prime}}{T}+\beta \frac{f^{\prime}}{f}+\gamma \frac{C^{\prime}}{C}+\delta \frac{\rho}{\rho^{\prime}}<\alpha+\beta+\gamma+\delta
$$

So when the area is far from crowded, we will use Eq.10 to evaluate our solution. During our evaluation of our solution, if the inequality (Eq.10) is not set up, we should propose a new solution. In addition, determine the affection when there are light and heavy traffic, more autonomous vehicles, and the different proportions of conventional tollbooths, exact-change tollbooths, and electronic toll collection booths (ETC) are asked in the problems.

We plan to handle the tasks into the following steps:

1)Establish an index to evaluate the time of a car passing the toll plaza and verify the effectiveness of the chosen index. Try to find the main causes to the index, the main causes can help us propose solutions later. 
2)Similarly, establish an index to evaluate the safety of a car passing the toll plaza and verify the effectiveness of the chosen index. Then find the main causes to the index, the main causes also can help us propose solutions later.

3)Establish the comprehensive evaluation model to verify the superiority of a new solution compared with the common toll plaza schemes. The comprehensive evaluation model is based on the indexes we find before.

4)Propose our solution by solving the most important factors found before and verify the superiority of our solution. Moreover, we need to analyze our solution performance in light and heavy traffic, and different proportion of ETC (electronic toll collection) booths. And analyze the sensitivity of some important indexes.

\section{Summary}

In this paper, a comprehensive evaluation model is proposed to determine if our solution is better than any common toll plaza. Using our comprehensive evaluation model, each decision maker may lay emphasis on the different index, so they can give the different weights. That means our model can be applied extensively.

\section{References}

[1]. M. Zhang, H. Chen, W.X. Wu. "Safety Evaluation Model for Expressway Toll Stations". China Safety Science Journal. Vol.19(2006)No.10, p. 139-144.

[2]. Gans, Noah, Mandelbaum A, et al. "Service times in call centers: Agent heterogeneity and learning with some operational consequences". Journal of Immunology .161.11(2010), p. 99-123.

[3]. C.L.Wu, Y.L.Chang. "Configuration Research of Toll-gate on Freeway”. Highway Engineering. Vol.33(2008)

[4]. No.5, p. 172-175.

[5]. H. Zhang."Empirical Analysis and Modeling of Manual Turnpike Tollbooths in China". Mechanical Systems and Signal Process. Elsevier 87(2017), p. 184-194. 